\title{
Sample Paths Properties of Stochastic Processes from Orlicz Spaces, with Applications to Partial Differential Equations
}

\author{
O. Hopkalo $^{1}$, Yu. Kozachenko ${ }^{1}$, E. Orsingher ${ }^{2}$ and L. Sakhno ${ }^{1, *}$ \\ ${ }^{1}$ Department of Probability Theory, Statistics and Actuarial Mathematics, Faculty of Mechanics and Mathematics, Taras Shevchenko \\ National University of Kyiv, Ukraine \\ ${ }^{2}$ Department of Statistical Sciences, Sapienza University of Rome, Italy
}

\begin{abstract}
In this paper we obtain conditions for stochastic processes from Orlicz spaces defined on unbounded domains to have almost sure bounded and continuous sample paths. Estimates for distributions of suprema of the processes are presented. Conditions are given in terms of entropy integrals and majorant characteristics of Orlicz spaces. Possible applications to solutions of partial differential equations are discussed. Examples of processes are given for which the conditions of the main results are satisfied.
\end{abstract}

Keywords stochastic processes from Orlicz spaces, distribution of supremum, continuity, boundedness, entropy methods, partial differential equations, random initial conditions.

AMS 2010 subject classifications 60G07, 60G17, 60G20, 35R60.

DOI: $10.19139 /$ soic-2310-5070-880

\section{Introduction}

In this paper, we apply entropy methods to studying sample paths of stochastic processes $X(t), t \in T$, where $T$ is a parameter set, belonging to Orlicz spaces of random variables. Recall that the entropy approach requires to evaluate entropy characteristics of the set $T$ with respect to particular metrics generated by the underlying process $X$. Then one can express assumptions on $X$ to be sample bounded, or to be sample continuous, or to have other properties, in terms of the so-called entropy integrals.

The origins of this approach can be traced back to the paper by Dudley [7], where sufficient conditions for the boundedness of Gaussian processes were based on the corresponding entropy integrals. Ideas from [7] were extended by Fernique in [9] and Ledoux and Talagrand in [16] using the majorising measures methods. Further studies by the mentioned authors and numerous other researchers resulted in a rich and well-developed theory providing tools for bounding Gaussian and related processes and treatment of other properties of their sample paths. A thorough presentation of these topics can be found, for example, in the classical monographs [1], [8], [17], [21], [22], see also references therein. We mention also the detailed account of conditions of regularity of sample paths of Gaussian processes given in [2, Section 3]. The questions of applicability of entropy based methods for more general classes of processes were treated by Buldygin and Kozachenko [4]. Section 2 in [4] is devoted to the full investigation of sample paths properties for stochastic processes taking values in Orlicz spaces of random variables. More specific attention is paid to a subclass of processes from Orlicz spaces of exponential type, which are called $\varphi$-sub-Gaussian processes and go beyond the Gaussian and sub-Gaussian ones.

\footnotetext{
*Correspondence to: L. Sakhno (lms@univ.kiev.ua), Department of Probability Theory, Statistics and Actuarial Mathematics, Faculty of Mechanics and Mathematics, Taras Shevchenko National University of Kyiv, 60 Volodymyrska str., 01601 Kyiv, Ukraine
}

ISSN 2310-5070 (online) ISSN 2311-004X (print)

Copyright (C) 2020 International Academic Press 
In this paper, our interest is focused on the case where the parameter set $T$ is unbounded. We investigate which conditions are required in order for the process $X$ to be sample bounded and sample continuous. We also obtain bounds for the distribution of the supremum of such processes. Note that the previous studies in [4, Chapter 3], [5], [12], [13], [15] considered these questions for processes defined on compact sets.

We cite some publications related to our approach. In [12], [13], the rate of growth of processes from Orlicz spaces over unbounded domains was studied and applied to wavelet expansions of such processes. Papers [6], [10] stated results on asymptotic bounds with probability 1 for the rate of growth of Gaussian processes defined on unbounded domains. These results were applied to problems of statistical estimation of models involving fractional Brownian motion. In [14] similar results for $\varphi$-sub-Gaussian processes were obtained. In [11], the rate of growth was evaluated for the solutions to higher-order heat-type equations with $\varphi$-sub-Gaussian initial conditions.

Our study of conditions for sample boundedness and sample continuity with probability 1 for stochastic processes is partially motivated by aplications to the study of Cauchy problems for partial differential equations with random initial conditions. Indeed, suppose we have properties of sample paths with probability 1 for a stochastic process taken as initial condition. If these properties correspond to those needed in nonrandom case, then we can apply the standard theory to study solutions of partial differential equations with such random initial condition. This is one of the possible approaches in the treatment of Cauchy problems with random initial conditions.

The structure of the paper is as follows. In Section 1 we present basic facts and definitions on Orlicz spaces of random variables and processes. In Section 2 we review the results on boundedness, continuity and the distribution of suprema of Orlicz processes defined on compact sets. On the base of the results of Section 2 we obtain the corresponding results for the processes defined on an unbounded domain in Section 3. Having stated the conditions of sample boundedness and continuity with probability 1 for Orlicz processes, we can use such processes to construct the models of processes related to some partial differential equations. Possible applications in this direction are discussed in Section 4. In Section 5, we present examples of the processes, which satisfy the conditions of theorems stated in Section 3.

\section{Orlicz spaces of random variables and processes. Basic definitions}

We present here some basic facts from the theory of Orlicz random variables and processes following the exposition in book [4, Chapter 2].

Definition 1.1. A continuous symmetric function $U=(U(x), x \in \mathbb{R})$, is called a $C$-function if $U(x)$ is monotone increasing for $x>0$ and $U(0)=0$.

Definition 1.2. We say that a $C$-function $U$ satisfies the $g$-condition if there exist constants $z_{0} \geq 0, K>0$, and $A>0$ such that the inequality

$$
U(x) U(y) \leq A U(K x y)
$$

holds for all $x \geq z_{0}, y \geq z_{0}$.

Example 1.3. The following functions are $C$-functions satisfying the $g$-condition:

1) For $a>0$ and $\alpha \geq 1$, the function $U(x)=a|x|^{\alpha}, x \in \mathbb{R}$, satisfies the $g$-condition with $K=1, A=a$, and $z_{0}=0$; 2) For $c>0, a>0$ and $\alpha \geq 1$, the function $U(x)=c\left(\exp \left\{a|x|^{\alpha}\right\}-1\right), x \in \mathbb{R}$, satisfies the $g$-condition with $K=1, A=c$, and $z_{0}=2^{1 / \alpha}$;

3) For $c>0$, the function $U(x)=c(\exp \{\phi(x)\}-1), x \in \mathbb{R}$, where $\phi(x), x \in \mathbb{R}$, is an arbitrary $C$-function, satisfies the $g$-condition with $K=1, A=c^{2}$, and $z_{0}=2$.

Definition 1.4. Let $(\Omega, \mathrm{F}, P)$ be a probability space, $U$ be an arbitrary $C$-function. The Orlicz space of random variables $L_{U}(\Omega)$ is defined as the family of random variables where for each $\xi \in L_{U}(\Omega)$ there exists a constant $r_{\xi}>0$ such that

$$
\mathrm{E} U\left(\frac{\xi}{r_{\xi}}\right)<\infty
$$


The Orlicz space $L_{U}(\Omega)$ is a Banach space with the norm

$$
\|\xi\|_{U}=\inf \left\{r>0: \mathrm{E} U\left(\frac{\xi}{r}\right) \leq 1\right\}
$$

which is called the Luxemburg norm.

Example 1.5. Suppose that $U(x)=|x|^{p}, x \in \mathbb{R}, p \geq 1$. Then $L_{U}(\Omega)$ is the space $L_{p}(\Omega)$, and the Luxemburg norm $\|\xi\|_{U}$ coincides with the norm $\|\xi\|_{p}=\left[\mathrm{E}|\xi|^{p}\right]^{1 / p}$.

The most useful property of Orlicz random variables is the estimate for their tail probabilities stated in the next lemma (see, [4, Lemma 2.3.1]).

Lemma 1.6. Assume that $U$ is an arbitrary $C$-function and take $\xi \in L_{U}(\Omega)$ satisfying $\|\xi\|_{U} \neq 0$. Then for all $x>0$

$$
\mathrm{P}\{|\xi|>x\} \leq\left(U\left(\frac{x}{\|\xi\|_{U}}\right)\right)^{-1} .
$$

Note: Here and in what follows we write $(U(\cdot))^{-1}$ for $\frac{1}{U(\cdot)}$, and the notation $U^{(-1)}(\cdot)$ stands for the inverse function to $U(\cdot)$.

Definition 1.7. A stochastic process $X(t), t \in T$, is called an Orlicz process belonging to the space $L_{U}(\Omega)$ if, for all $t \in T$, the random variables $X(t)$ belong to $L_{U}(\Omega)$.

The following notion is important for studying properties of sample paths of Orlicz processes.

Definition 1.8. A monotone non decreasing sequence of positive numbers $\left(\chi_{U}(n), n \in \mathbb{N}\right)$ is called the $M$ characteristic (majorant characteristic) of an Orlicz space if for any $n \in \mathbb{N}$ and $\xi_{k} \in L_{U}(\Omega), k=1, \ldots, n$, the following inequality holds:

$$
\left\|\max _{1 \leq k \leq n}\left|\xi_{k}\right|\right\|_{U} \leq \chi_{U}(n) \max _{1 \leq k \leq n}\left\|\xi_{k}\right\|_{U}
$$

Definition 1.8 enables us to control an Orlicz norm of the maximum of a family of random variables belonging to an Orlicz space by means of the $M$-characteristic of this space and using information (for example, upper bounds) on individual Orlicz norms of the members of this family of random variables.

For a function $U$ satisfying the $g$-condition, Lemma 1.9 expresses the $M$-characteristic of the space $L_{U}(\Omega)$ in a form that is convenient for applications.

Lemma 1.9. Assume that a function $U$ satisfies the $g$-condition and $U^{(-1)}$ is the inverse function to $U$. Take $z_{0} \geq 0$, $A>0$ and $K>0$ appearing in Definition 1.2. Set

$$
C_{U}=K\left(1+U\left(z_{0}\right) \max (1, A)\right) .
$$

and define a sequence $\left(\chi_{U}(n), n \in \mathbb{N}\right)$ in the following way:

$$
\chi_{U}(n)= \begin{cases}C_{U} U^{(-1)}(n), & \text { if } n>U\left(z_{0}\right) \\ n, & \text { if } n \leq U\left(z_{0}\right)\end{cases}
$$

Then $\left(\chi_{U}(n), n \in \mathbb{N}\right)$ is the $M$-characteristic of the space $L_{U}(\Omega)$.

As we can see, for a function $U$ satisfying the $g$-condition, the Orlicz norm of the maximum of $n$ variables behaves as $U^{(-1)}(n), n \in \mathbb{N}$.

Example 1.10. If $U(x)=|x|^{p}, x \in \mathbb{R}$, for some $p \geq 1$, then $\chi_{U}(n)=n^{1 / p}, n \in \mathbb{N}$. 


\section{Sample paths properties of Orlicz processes defined on a compact}

In this section, we review some results for Orlicz processes which will be used to state the results in Section 3.

Let $X=\{X(t) ; t \in T\}$ be an Orlicz stochastic process, $(T, \rho)$ be a metric (pseudometric) space. Recall that a function $\rho: T \times T \rightarrow[0, \infty)$ is a pseudometric on $T$ if it satisfies all properties of a metric but with one exception: if $\rho(t, s)=0$, then not necessarily $t=s$.

Let $N(u)=N_{\rho}(T, u), u>0$, be the metric massiveness of the set $T$ with respect to the pseudometric $\rho$, that is, the smallest number of closed balls of a radius at most equal to $u$ necessary to cover $T$.

The notions of the metric massiveness $N(u), u>0$, or the metric entropy $H(u):=\ln N(u), u>0$, are useful for describing the properties of sample paths of the process $X$.

Entropy methods enable us to study whether a process belonging to an Orlicz space $L_{U}(\Omega)$ is sample bounded and sample continuous. The distribution of the supremum of a process in this space can be expressed in terms of the so-called entropy integrals involving the metric massiveness $N$ and the $M$-characteristic $\chi_{U}$ of the underlying Orlicz space.

The most general bound for the norm of the supremum of a process belonging to an Orlicz space $L_{U}(\Omega)$, which entails also an estimate for the distribution of the supremum itself, was stated in book [4, Theorem 3.3.2]. In [12] this result was specified under an additional condition on the process increments.

Theorem 2.1 ([12], Theorem 2.2). Let (T, $\rho)$ be a compact metric (pseudometric) space and denote by $N$ the metric massiveness of $(T, \rho)$. Let $X=\{X(t) ; t \in T\}$ be a separable stochastic process from an Orlicz space $L_{U}(\Omega)$ and

$$
\sup _{\rho(t, s) \leq h}\|X(t)-X(s)\|_{U} \leq \sigma(h)
$$

where $\sigma=\left\{\sigma(h), 0 \leq h \leq \sup _{t, s \in T} \rho(t, s)\right\}$ is a monotonically increasing continuous function and $\sigma(0)=0$.

Suppose that for some $\varepsilon>0$

$$
\int_{0}^{\varepsilon} \chi_{U}\left(N\left(\sigma^{(-1)}(u)\right)\right) d u<\infty
$$

where the function $\sigma^{(-1)}(u)$ is the inverse of the function $\sigma(u),\left(\chi_{U}(n), n \in \mathbb{N}\right)$ is the M-characteristic of the space $L_{U}(\Omega)$. Then with probability 1 the random variable $\sup _{t \in T}|X(t)|$ belongs to the space $L_{U}(\Omega)$, and

$$
\left\|\sup _{t \in T}|X(t)|\right\|_{U} \leq\left\|X\left(t_{0}\right)\right\|_{U}+\frac{1}{\theta(1-\theta)} \int_{0}^{\omega_{0} \theta} \chi_{U}\left(N\left(\sigma^{(-1)}(u)\right)\right) d u=: B\left(t_{0}\right),
$$

where $t_{0} \in T$ is an arbitrary point, $\omega_{0}=\sigma\left(\sup _{t \in T} \rho\left(t_{0}, t\right)\right), 0<\theta<1$.

In addition, for any $\varepsilon>0$ the following inequality holds:

$$
\mathrm{P}\left\{\sup _{t \in T}|X(t)|>\varepsilon\right\} \leq\left(U\left(\frac{\varepsilon}{B\left(t_{0}\right)}\right)\right)^{-1} .
$$

The next theorem is a variant of a general result stated in [4, Theorem 3.5.2].

Theorem 2.2 ([12], Theorem 2.3). Let $(T, \rho)$ be a compact metric (pseudometric) space and let $X$ be a separable stochastic process belonging to an Orlicz space $L_{U}(\Omega)$. Suppose that the assumptions in Theorem 2.1 hold. Then we have

$$
\lim _{h \rightarrow 0}\left\|\sup _{\rho(t, s) \leq h}|X(t)-X(s)|\right\|_{U}=0
$$

and the process $X$ is sample continuous on $(T, \rho)$ with probability 1 .

Introducing the additional assumption on the function $U$, the above theorems can be reformulated in the form, which is more convenient for applications. 
Theorem 2.3. Let $(T, \rho)$ be a compact metric (pseudometric) space, $N$ be the metric massiveness of $(T, \rho)$. Let $X=\{X(t) ; t \in T\}$ be a separable stochastic process from an Orlicz space $L_{U}(\Omega)$ with a function $U$ satisfying the $g$-condition, and

$$
\sup _{\rho(t, s) \leq h}\|X(t)-X(s)\|_{U} \leq \sigma(h)
$$

where $\sigma=\left\{\sigma(h), 0 \leq h \leq \sup _{t, s \in T} \rho(t, s)\right\}$ is a monotonically increasing continuous function and $\sigma(0)=0$.

Suppose that for some $\varepsilon>0$

$$
\int_{0}^{\varepsilon} U^{(-1)}\left(N\left(\sigma^{(-1)}(u)\right)\right) d u<\infty .
$$

Then with probability 1 the random variable $\sup _{t \in T}|X(t)|$ belongs to the space $L_{U}(\Omega)$, and

$$
\left\|\sup _{t \in T}|X(t)|\right\|_{U} \leq\left\|X\left(t_{0}\right)\right\|_{U}+\frac{C_{U}}{\theta(1-\theta)} \int_{0}^{\omega_{0} \theta} U^{(-1)}\left(N\left(\sigma^{(-1)}(u)\right)\right) d u=: D\left(t_{0}\right),
$$

where $t_{0} \in T$ is an arbitrary point, $\omega_{0}=\sigma\left(\sup _{t \in T} \rho\left(t_{0}, t\right)\right), C_{U}$ is given by (1.1), $\theta$ is an arbitrary point such that $0<\theta<\min (1, \hat{\theta})$ for $\hat{\theta}=\sup \left\{\theta>0: N\left(\sigma^{(-1)}\left(\omega_{0} \theta\right)\right) \geq U\left(z_{0}\right)\right\}$.

In addition, for any $\varepsilon>0$ the following inequality holds:

$$
\mathrm{P}\left\{\sup _{t \in T}|X(t)|>\varepsilon\right\} \leq\left(U\left(\frac{\varepsilon}{D\left(t_{0}\right)}\right)\right)^{-1},
$$

and the process $X$ is sample continuous on $(T, \rho)$ with probability 1 .

Proof

The theorem follows from Theorems 2.1 and 2.2. A variant of this theorem was given in [15, Theorem 3.4]. The restriction on $\theta$ can be deduced from the expression for $\chi_{U}$. Indeed, in our case we have $\chi_{U}(n)=C_{U} U^{(-1)}(n)$ for $n>U\left(z_{0}\right)$ (Lemma 1.9) and

$$
\chi_{U}\left(N\left(\sigma^{(-1)}(u)\right)=C_{U} U^{(-1)}\left(N\left(\sigma^{(-1)}(u)\right)\right.\right.
$$

for $N\left(\sigma^{(-1)}(u) \geq U\left(z_{0}\right)\right.$, and since $0<u<\theta \omega_{0}$, we obtain that $\theta$ should be such that

$$
N\left(\sigma^{(-1)}\left(\theta \omega_{0}\right) \geq U\left(z_{0}\right) .\right.
$$

The fact that $X$ is sample continuous follows from Theorem 2.2.

Consider now the space $\mathbb{R}^{n}$ with the metric

$$
\hat{\rho}(\bar{t}, \bar{s})=\max _{i=1, n}\left|t_{i}-s_{i}\right|, \quad \bar{t}=\left(t_{1}, \ldots, t_{n}\right), \quad \bar{s}=\left(s_{1}, \ldots, s_{n}\right),
$$

and the compact set $\hat{T}=\left(a_{i} \leq t_{i} \leq b_{i}, i=\overline{1, n}\right)$, for some $\bar{a}, \bar{b} \in \mathbb{R}^{n}, a_{i}<b_{i}, i=1, \ldots, n$.

For the metric massiveness $N$ of the set $\hat{T}$ with respect to metric $\hat{\rho}$, the following inequality holds:

$$
\prod_{i=1}^{n}\left(\frac{b_{i}-a_{i}}{2 u}\right) \leq N(u) \leq \prod_{i=1}^{n}\left(\frac{b_{i}-a_{i}}{2 u}+1\right), u>0,
$$

and inequality (2.7) will be true if

$$
\prod_{i=1}^{n}\left(\frac{b_{i}-a_{i}}{2 \sigma^{(-1)}\left(\theta \omega_{0}\right)}\right) \geq U\left(z_{0}\right)
$$

Hence, we have the following result as a corollary of Theorem 2.3. 
Theorem 2.4. Let $\hat{T}=\left(a_{i} \leq t_{i} \leq b_{i}, i=\overline{1, n}\right)$ with metric $\hat{\rho}(\bar{t}, \bar{s})$ defined by (2.8), $X=(X(\bar{t}), \bar{t} \in \hat{T})$ be a separable stochastic process from an Orlicz space $L_{U}(\Omega)$ with a function $U(x)$ satisfying the g-condition, and

$$
\sup _{\hat{\rho}(\bar{t}, \bar{s}) \leq h}\|X(\bar{t})-X(\bar{s})\| \leq \sigma(h),
$$

where $\sigma=\left\{\sigma(h), 0 \leq h \leq \sup _{\bar{t}, \bar{s} \in \hat{T}} \hat{\rho}(\bar{t}, \bar{s})\right\}$ is a monotonically increasing continuous function and $\sigma(0)=0$.

Suppose that for some $\varepsilon>0$

$$
\int_{0}^{\varepsilon} U^{(-1)}\left(\prod_{i=1}^{n}\left(\frac{b_{i}-a_{i}}{2 \sigma^{(-1)}(u)}+1\right)\right) d u<\infty
$$

Then with probability 1 the random variable $\sup _{\bar{t} \in \hat{T}}|X(\bar{t})|$ belongs to the space $L_{U}(\Omega)$, and

$$
\left\|\sup _{\bar{t} \in \hat{T}}|X(\bar{t})|\right\|_{U} \leq\left\|X\left(\overline{t_{0}}\right)\right\|_{U}+\frac{C_{U}}{\theta(1-\theta)} \int_{0}^{\hat{\omega}_{0} \theta} U^{(-1)}\left(\prod_{i=1}^{n}\left(\frac{b_{i}-a_{i}}{2 \sigma^{(-1)}(u)}+1\right)\right) d u=: R\left(\bar{t}_{0}\right),
$$

where $\bar{t}_{0} \in \hat{T}$ is an arbitrary point, $\hat{\omega}_{0}=\sigma\left(\sup _{\bar{t} \in \hat{T}} \hat{\rho}\left(\bar{t}_{0}, \bar{t}\right)\right), C_{U}$ is given by (1.1), $\theta$ is an arbitrary point such that

$$
0<\theta<\min \left(1, \frac{1}{\hat{\omega}_{0}} \sigma\left(\frac{1}{2}\left(\frac{\prod_{i=1}^{n}\left(b_{i}-a_{i}\right)}{U\left(z_{0}\right)}\right)^{1 / n}\right)\right) .
$$

In addition, for any $\varepsilon>0$ the following inequality holds:

$$
\mathrm{P}\left\{\sup _{\bar{t} \in \hat{T}}|X(\bar{t})|>\varepsilon\right\} \leq\left(U\left(\frac{\varepsilon}{R\left(\bar{t}_{0}\right)}\right)\right)^{-1} .
$$

and the process $X$ is sample continuous on $(\hat{T}, \hat{\rho})$ with probability 1.

\section{Orlicz processes defined on non-compact metric spaces}

In this section we consider the case of a space $(T, \rho)$, where $T$ is not compact, but is $\sigma$-compact, that is, $T$ can be represented as a countable union of compact subsets. As we have seen in the previous section, to state the boundedness and continuity of Orlicz processes on compact sets we can apply the same conditions formulated in terms of entropy integrals. For non-compact sets the situation is different. The study of continuity is a simpler task: if $T$ is a $\sigma$-compact, then almost sure continuity of the process on compact subsets of $T$ (which holds under finiteness of corresponding entropy integrals on these subsets) will entail almost sure continuity on the whole $T$ (see, e.g., [3, Section 1.3]). But the same arguments do not hold, if we want to state the boundedness of a process, and some additional assumptions are needed. One possible way to solve this problem is presented in the next theorem.

Theorem 3.1. Let $(T, \rho)$ be a non-compact metric space and there exist compact sets $\left(T_{k}, \rho\right), k \in \mathbb{Z}$, such that $(T, \rho)=\bigcup_{k \in \mathbb{Z}}\left(T_{k}, \rho\right)$. Let $N_{\rho}\left(T_{k}, u\right), u>0$, be the metric massiveness of the compact set $T_{k}$ with respect to the pseudometric $\rho,\left(\chi_{U}(n), n \in \mathbb{N}\right)$ be the M-characteristic of the Orlicz space $L_{U}(\Omega)$. Suppose that $X=\{X(t) ; t \in$ $T\}$ is a separable stochastic process from an Orlicz space $L_{U}(\Omega)$ and there exist monotonically increasing continuous functions $\sigma_{k}=\left\{\sigma_{k}(h), 0 \leq h \leq \max _{t, s \in T_{k}} \rho(t, s)\right\}, k \in \mathbb{Z}$, such that $\sigma_{k}(0)=0$ and

$$
\sup _{\rho(t, s) \leq h, t, s \in T_{k}}\|X(t)-X(s)\|_{U} \leq \sigma_{k}(h) .
$$

Suppose that for some $\varepsilon>0$, for all $k \in \mathbb{Z}$

$$
\int_{0}^{\varepsilon} \chi_{U}\left(N_{\rho}\left(T_{k}, \sigma_{k}^{(-1)}(u)\right)\right) d u<\infty .
$$


Let

$$
B_{t_{0 k}}=\left\|X\left(t_{0 k}\right)\right\|_{U}+\frac{1}{\theta(1-\theta)} \int_{0}^{\omega_{0 k} \theta} \chi_{U}\left(N_{\rho}\left(T_{k}, \sigma_{k}^{(-1)}(u)\right)\right) d u,
$$

where $t_{0 k} \in T_{k}, \omega_{0 k}=\sigma_{k}\left(\sup _{t \in T_{k}} \rho\left(t_{0 k}, t\right)\right), 0<\theta<1$. If for some $t_{0 k}$ and $0<\theta<1$, for all $\varepsilon>0$ the series

$$
\sum_{k \in \mathbb{Z}}\left(U\left(\frac{\varepsilon}{B_{t_{0 k}}}\right)\right)^{-1}
$$

is convergent, then

$$
\mathrm{P}\left\{\sup _{t \in T}|X(t)|>\varepsilon\right\} \leq \sum_{k \in \mathbb{Z}}\left(U\left(\frac{\varepsilon}{B\left(t_{0 k}\right)}\right)\right)^{-1}
$$

and with probability 1

$$
\sup _{t \in T}|X(t)|<\infty
$$

and the process $X$ is almost surely sample continuous on $(T, \rho)$.

Proof

The proof of the theorem follows from Theorem 2.1 and from the fact, that for $\varepsilon>0$

$$
\mathrm{P}\left\{\sup _{t \in T}|X(t)|>\varepsilon\right\} \leq \sum_{k \in \mathbb{Z}} \mathrm{P}\left\{\sup _{t \in T_{k}}|X(t)|>\varepsilon\right\} .
$$

Convergence of the series in the right hand side of (3.6) for all $\varepsilon>0$ implies the boundedness of the process $X$ on $T$ with probability 1 .

Similarly, from Theorems 2.3 and 2.4 the next two theorems follow.

Theorem 3.2. Let $(T, \rho)$ be a non-compact metric space, $X=\{X(t) ; t \in T\}$ be a separable stochastic process from an Orlicz space $L_{U}(\Omega)$ with a function $U$ satisfying the g-condition. Suppose that there exist compact sets $\left(T_{k}, \rho\right), k \in \mathbb{Z}$, such that $(T, \rho)=\bigcup_{k \in \mathbb{Z}}\left(T_{k}, \rho\right)$. Let $N_{\rho}\left(T_{k}, u\right), u>0$ be the metric massiveness of the compact set $T_{k}$ with respect to the pseudometric $\rho$. Suppose that there exist monotonically increasing continuous functions $\sigma_{k}=\left\{\sigma_{k}(h), 0 \leq h \leq \max _{t, s \in T_{k}} \rho(t, s)\right\}, k \in \mathbb{Z}$, such that $\sigma_{k}(0)=0$ and

$$
\sup _{\rho(t, s) \leq h, t, s \in T_{k}}\|X(t)-X(s)\|_{U} \leq \sigma_{k}(h) .
$$

Suppose that for some $\varepsilon>0$, for all $k \in \mathbb{Z}$

$$
\int_{0}^{\varepsilon} U^{(-1)}\left(N_{\rho}\left(T_{k}, \sigma_{k}^{(-1)}(u)\right)\right) d u<\infty .
$$

Define for each $k \in \mathbb{Z}$

$$
D\left(t_{0 k}\right)=\left\|X\left(t_{0 k}\right)\right\|_{U}+\frac{C_{U}}{\theta(1-\theta)} \int_{0}^{\omega_{0 k} \theta} U^{(-1)}\left(N_{\rho}\left(T_{k}, \sigma_{k}^{(-1)}(u)\right)\right) d u,
$$

where $t_{0 k} \in T_{k}, \omega_{0 k}=\sigma_{k}\left(\sup _{t \in T_{k}} \rho\left(t_{0 k}, t\right)\right), C_{U}$ is given by (1.1), $\theta$ is an arbitrary point such that $0<\theta<$ $\min \left(1 ; \hat{\theta}_{k}\right)$ for $\hat{\theta}_{k}=\sup \left\{\theta>0: N_{\rho}\left(T_{k}, \sigma_{k}^{(-1)}\left(\omega_{0 k} \theta\right)\right) \geq U\left(z_{0}\right)\right\}$.

If for some $t_{0 k}$ and $\theta$ such that $0<\theta<\min _{k \in \mathbb{Z}}\left(1, \hat{\theta}_{k}\right)$, and for all $\varepsilon>0$ the series

$$
\sum_{k \in \mathbb{Z}}\left(U\left(\frac{\varepsilon}{D_{t_{0 k}}}\right)\right)^{-1}
$$


is convergent, then

$$
\mathrm{P}\left\{\sup _{t \in T}|X(t)|>\varepsilon\right\} \leq \sum_{k \in \mathbb{Z}}\left(U\left(\frac{\varepsilon}{D\left(t_{0 k}\right)}\right)\right)^{-1},
$$

and with probability 1

$$
\sup _{t \in T}|X(t)|<\infty
$$

and the process $X$ is almost surely sample continuous on $(T, \rho)$.

Corollary 3.3. Let under the conditions of Theorem 3.2, $\sigma_{k}(h)=Z\left(T_{k}\right) \sigma(h), h \geq 0$, where $Z\left(T_{k}\right)>0, k \in \mathbb{Z}$, are some constants depending only on $T_{k}$. Then we can replace the condition (3.8) by the following one:

$$
I_{k}(\varepsilon)=\int_{0}^{\varepsilon} U^{(-1)}\left(N_{\rho}\left(T_{k}, \sigma^{(-1)}(u)\right)\right) d u<\infty,
$$

and the expression for $D\left(t_{0 k}\right)$ takes the form

$$
D\left(t_{0 k}\right)=\left\|X\left(t_{0 k}\right)\right\|_{U}+\frac{C_{U}}{\theta(1-\theta)} Z\left(T_{k}\right) I_{k}\left(\omega_{0 k} \theta\right)
$$

where $\omega_{0 k}=\sigma\left(\sup _{t \in T_{k}} \rho\left(t_{0 k}, t\right)\right)$, and $\theta$ is an arbitrary point such that $0<\theta<\min (1, \hat{\theta})$ with $\hat{\theta}=\sup \{\theta>0$ : $\left.N_{\rho}\left(T_{k}, Z\left(T_{k}\right) \sigma^{(-1)}\left(\omega_{0 k} \theta\right)\right) \geq U\left(z_{0}\right)\right\}$.

If the series (3.10) with $D\left(t_{0 k}\right)$ given by (3.11) is convergent, then the statements of Theorem 3.2 hold true.

Proof

Indeed, since $\sigma_{k}^{(-1)}(u)=\sigma^{(-1)}\left(\frac{u}{Z\left(T_{k}\right)}\right)$, we obtain

$$
\int_{0}^{\varepsilon} U^{(-1)}\left(N_{\rho}\left(T_{k}, \sigma_{k}^{(-1)}(u)\right)\right) d u=Z\left(T_{k}\right) \int_{0}^{\varepsilon / Z\left(T_{k}\right)} U^{(-1)}\left(N_{\rho}\left(T_{k}, \sigma^{(-1)}(u)\right)\right) d u<\infty,
$$

and the expression for $D\left(t_{0 k}\right)$ should be modified correspondingly.

We now specify the general results for the particular case of the space $\mathbb{R}^{n}$, which will be convenient for applications.

Theorem 3.4. Let $X=\left\{X(\bar{t}) ; \bar{t} \in \mathbb{R}^{n}\right\}$ be a separable stochastic process belonging to an Orlicz space $L_{U}(\Omega)$, with the function $U(x)$ satisfying the $g$-condition. Let $\hat{T}_{k}, k \in \mathbb{Z}$, be of the form $\hat{T}_{k}=\left\{a_{k, i} \leq t_{i} \leq a_{k+1, i}, i=\overline{1, n}\right\}$, for $a_{k, i} \in \mathbb{R}, a_{k, i}<a_{k+1, i}, k \in \mathbb{Z}, i=\overline{1, n}$, and such that $\mathbb{R}^{n}=\bigcup_{k \in \mathbb{Z}} \hat{T}_{k}$. Choose on $\mathbb{R}^{n}$ the metric $\hat{\rho}(\bar{t}, \bar{s})=$ $\max _{i=1, \ldots, n}\left|t_{i}-s_{i}\right|$, where $\bar{t}=\left(t_{1}, \ldots, t_{n}\right), \bar{s}=\left(s_{1}, \ldots, s_{n}\right)$.

Suppose that there exist monotonically increasing continuous functions

$\sigma_{k}=\left\{\sigma_{k}(h), 0 \leq h \leq \max _{\bar{t}, \bar{s} \in \hat{B}_{k}} \rho(\bar{t}, \bar{s})\right\}, \sigma_{k}(0)=0$, such that for all $k \in \mathbb{Z}$

$$
\sup _{\rho(\bar{t}, \bar{s}) \leq h, \bar{t}, \bar{s} \in \hat{T}_{k}}\|X(\bar{t})-X(\bar{s})\|_{U} \leq \sigma_{k}(h) .
$$

Suppose that for some $\varepsilon>0$, for all $k \in \mathbb{Z}$

$$
\int_{0}^{\varepsilon} U^{(-1)}\left(\prod_{i=1}^{n}\left(\frac{a_{k+1, i}-a_{k, i}}{2 \sigma_{k}^{(-1)}(u)}+1\right)\right) d u<\infty
$$

Let

$$
R\left(\bar{t}_{0 k}\right)=\left\|X\left(\bar{t}_{0 k}\right)\right\|_{U}+\frac{1}{\theta(1-\theta)} \int_{0}^{\omega_{0 k} \theta} U^{(-1)}\left(\prod_{i=1}^{n}\left(\frac{a_{k+1, i}-a_{k, i}}{2 \sigma_{k}^{(-1)}(u)}+1\right)\right) d u,
$$


where $\bar{t}_{0 k} \in \hat{T}_{k}$ is an arbitrary point, $\omega_{0 k}=\sigma_{k}\left(\sup _{\bar{t} \in \hat{T}_{k}} \rho\left(\bar{t}_{0 k}, \bar{t}\right)\right), \theta$ is an arbitrary point such that $0<\theta<1$. Iffor some $\bar{t}_{0 k}, \theta$, such that $0<\theta<1$, and $\varepsilon>0$ the series

$$
\sum_{k \in \mathbb{Z}}\left(U\left(\frac{\varepsilon}{R\left(\bar{t}_{0 k}\right)}\right)\right)^{-1}
$$

is convergent, then

$$
\mathrm{P}\left\{\sup _{\bar{t} \in \mathbb{R}^{n}}|X(\bar{t})|>\varepsilon\right\} \leq \sum_{k \in \mathbb{Z}}\left(U\left(\frac{\varepsilon}{R\left(\bar{t}_{0 k}\right)}\right)\right)^{-1}
$$

with probability 1

$$
\sup _{\bar{t} \in \mathbb{R}^{n}}|X(\bar{t})|<\infty
$$

and the process $X$ is almost surely sample continuous on $\mathbb{R}^{n}$.

We state now the corollary from the above theorem for the processes from the space $L_{p}(\Omega), p \geq 1$, which is the Orlicz space $L_{U}(\Omega)$ with $U(x)=|x|^{p}, x \in \mathbb{R}, p \geq 1$. This theorem will be used to construct examples in Section 5. In this case the conditions appear in the form, which is convenient for calculations.

Theorem 3.5. Let $X=\left\{X(\bar{t}) ; \bar{t} \in \mathbb{R}^{n}\right\}$ be a separable stochastic process from the space $L_{p}(\Omega), p \geq 1$. Let $\hat{B}_{k}, k \in$ $\mathbb{Z}$, be sets of the form $\hat{B}_{k}=\left\{a_{k, i} \leq t_{i} \leq a_{k+1, i}, i=1, \ldots, n\right\}$ for $a_{k, i} \in \mathbb{R}, a_{k, i}<a_{k+1, i}, k \in \mathbb{Z}, i=\overline{1, n}$, and such that $\mathbb{R}^{n}=\bigcup_{k \in \mathbb{Z}} \hat{B_{k}}$. Let the metric be defined as $\hat{\rho}(\bar{t}, \bar{s})=\max _{i=1, \ldots, n}\left|t_{i}-s_{i}\right|$, where $\bar{t}=\left(t_{1}, \ldots, t_{n}\right), \bar{s}=$ $\left(s_{1}, \ldots, s_{n}\right)$.

Suppose that there exist monotonically increasing continuous functions

$\sigma_{k}=\left\{\sigma_{k}(h), 0 \leq h \leq \max _{\bar{t}, \bar{s} \in \hat{B_{k}}} \rho(\bar{t}, \bar{s})\right\}, \sigma_{k}(0)=0$, such that on each set $\hat{B}_{k}$

$$
\sup _{\rho(\bar{t}, \bar{s}) \leq h, \bar{t}, \bar{s} \in \hat{B_{k}}}\|X(\bar{t})-X(\bar{s})\|_{L_{p}(\Omega)}=\sup _{\rho(\bar{t}, \bar{s}) \leq h, \bar{t}, \bar{s} \in \hat{B_{k}}}\left(\mathrm{E}|X(\bar{t})-X(\bar{s})|^{p}\right)^{\frac{1}{p}} \leq \sigma_{k}(h) .
$$

Suppose that for some $\varepsilon>0$, for all $k \in \mathbb{Z}$

$$
\int_{0}^{\varepsilon}\left|\prod_{i=1}^{n}\left(\frac{\left(a_{k+1, i}-a_{k, i}\right)}{2 \sigma_{k}^{(-1)}(u)}+1\right)\right|^{1 / p} d u<\infty
$$

Let

$$
R\left(\bar{t}_{0 k}\right)=\left(\mathrm{E}\left|X\left(\bar{t}_{0 k}\right)\right|^{p}\right)^{1 / p}+\frac{1}{\theta(1-\theta)} \int_{0}^{\omega_{0 k} \theta}\left|\prod_{i=1}^{n}\left(\frac{\left(a_{k+1, i}-a_{k, i}\right)}{2 \sigma_{k}^{(-1)}(u)}+1\right)\right|^{1 / p} d u,
$$

where $\bar{t}_{0 k} \in \hat{B_{k}}$ is an arbitrary point, $\omega_{0 k}=\sigma_{k}\left(\sup _{\bar{t} \in \hat{B_{k}}} \rho\left(\bar{t}_{0 k}, \bar{t}\right)\right), \theta$ is an arbitrary point, $0<\theta<1$.

If for some $\bar{t}_{0 k}$, for every $\theta$ such that $0<\theta<1$, and $\varepsilon>0$ the series

$$
\sum_{k \in \mathbb{Z}}\left(R\left(\bar{t}_{0 k}\right)\right)^{p}
$$

is convergent, then

$$
\mathrm{P}\left\{\sup _{\bar{t} \in \mathbb{R}^{n}}|X(\bar{t})|>\varepsilon\right\} \leq \frac{1}{\varepsilon^{p}} \sum_{k \in \mathbb{Z}}\left(R\left(\bar{t}_{0 k}\right)\right)^{p}
$$

with probability 1

$$
\sup _{\bar{t} \in \mathbb{R}^{n}}|X(\bar{t})|<\infty
$$

and the process $X$ is almost surely sample continuous. 
Corollary 3.6. Let under the conditions of Theorem 3.5, $\sigma_{k}(h)=r\left(\hat{B}_{k}\right) \sigma(h), h \geq 0$, where $r\left(\hat{B}_{k}\right) \geq 0, k \in \mathbb{Z}$, are some constants depending only on $\hat{B}_{k}$. Then we can replace the condition (3.16) in Theorem 3.5 by the following one:

$$
I_{k}(\varepsilon):=\int_{0}^{\varepsilon}\left|\prod_{i=1}^{n}\left(\frac{\left(a_{k+1, i}-a_{k, i}\right)}{2 \sigma_{k}^{(-1)}(u)}+1\right)\right|^{1 / p} d u<\infty,
$$

and the expression for $R\left(\bar{t}_{0 k}\right)$ takes the form

$$
R\left(\bar{t}_{0 k}\right)=\left(\mathrm{E}\left|X\left(\bar{t}_{0 k}\right)\right|^{p}\right)^{1 / p}+\frac{r\left(\hat{B}_{k}\right)}{\theta(1-\theta)} I_{k}\left(\omega_{0 k} \theta\right),
$$

where $\bar{t}_{0 k} \in \hat{B}_{k}$ is an arbitrary point, $\omega_{0 k}=\sigma\left(\sup _{\bar{t} \in \hat{B_{k}}} \rho\left(\bar{t}_{0 k}, \bar{t}\right)\right), \theta$ is an arbitrary point, $0<\theta<1$. If the series (3.17) with $R\left(\bar{t}_{0 k}\right)$ given by (3.18) is convergent, then the statements of Theorem 3.5 hold true.

In Section 5 we present examples of the processes for which conditions of the above theorems are satisfied.

\section{Applications to solutions of Cauchy problems for partial differential equations with random initial conditions}

In this section we discuss some applications of the results of Section 3.

Let $\xi(x), x \in \mathbb{R}^{n}$, be a sample bounded and sample continuous process with probability 1 . In particular, we assume that $\xi$ is a process belonging to an Orlicz space $L_{U}(\Omega)$ for which the conditions of Theorem 3.4 hold.

Consider the stochastic process $u(t, x), t \geq 0, x \in \mathbb{R}^{n}$, defined by the following integral

$$
\begin{aligned}
u(t, x) & =\int_{\mathbb{R}^{n}} p(t, y-x) \xi(y) d y, \\
u(0, x) & =\xi(x),
\end{aligned}
$$

where the kernel $p(t, x), t>0, x \in \mathbb{R}^{n}$, satisfies the conditions:

1) $p(t, x) \geq 0, t>0, x \in \mathbb{R}^{n}$;

2) for all $t>0$

$$
\int_{\mathbb{R}^{n}} p(t, x) d x=1
$$

3) for any bounded continuous function $f(x), x \in \mathbb{R}^{n}$,

$$
\lim _{t \rightarrow+0} \int_{\mathbb{R}^{n}} p(t, y-x) f(y) d y=f(x) .
$$

Theorems 3.4 and 3.5 give us the instruments to check whether a process $\xi$ from an Orlicz space $L_{U}(\Omega)$ is sample bounded and sample continuous. Therefore, basing on such $\xi$, the processes introduced by (4.19)-(4.20) will be well defined, and, moreover, their suprema can be estimated.

We get the following simple result.

Lemma 4.1. Let $\xi(x), x \in \mathbb{R}^{n}$, be an almost surely sample bounded and sample continuous process, and let the process $u(t, x), t \geq 0, x \in \mathbb{R}^{n}$, be defined by (4.19)-(4.20). Then with probability 1 the process $u(t, x)$ is bounded and

$$
|u(t, x)| \leq \sup _{x \in \mathbb{R}^{n}}|\xi(x)|, \quad \sup _{t \geq 0, x \in \mathbb{R}^{n}}|u(t, x)|=\sup _{x \in \mathbb{R}^{n}}|\xi(x)| .
$$

Proof

From the equality $u(0, x)=\xi(x)$ we have immediately that

$$
\sup _{x \in \mathbb{R}^{n}}|u(0, x)|=\sup _{x \in \mathbb{R}^{n}}|\xi(x)| .
$$


On the other hand, in view of properties of the process $\xi$ and the kernel function, we have for $t>0, x \in \mathbb{R}^{n}$

$$
|u(t, x)| \leq \int_{\mathbb{R}^{n}}|\xi(v)| p(t, v-x) d v \leq \sup _{v \in \mathbb{R}^{n}}|\xi(v)| \int_{\mathbb{R}^{n}} p(t, v-x) d v=\sup _{v \in \mathbb{R}^{n}}|\xi(v)|,
$$

therefore,

$$
\sup _{t>0, x \in \mathbb{R}^{n}}|u(t, x)| \leq \sup _{x \in \mathbb{R}^{n}}|\xi(x)| .
$$

The relations (4.22) and (4.23) imply the second equality in (4.21).

With a particular choice of the kernel $p$, the formulas (4.19)-(4.20) give a (space-time) process defined on $\left\{(t, x): t \geq 0, x \in \mathbb{R}^{n}\right\}, n \geq 1$, which can be treated as a solution to a Cauchy problem for a partial differential equation with the random initial condition given by $\xi$. If the sample paths of the process $\xi$ have properties with probability 1 , which coincide with those needed in the nonrandom case, then one can apply the standard results of theory of partial differential equations. We present below some examples of such kernels.

Let $p$ in (4.19) be the (multidimensional) heat kernel:

$$
p(t, x)=\frac{1}{(2 a \sqrt{\pi t})^{n}} \exp \left\{-\frac{|x|^{2}}{4 a^{2} t}\right\}, t>0, x \in \mathbb{R}^{n} .
$$

Consider the Cauchy problem for the heat equation

$$
\left\{\begin{array}{l}
u_{t}(t ; x)=a \Delta u(t ; x), t>0, x \in \mathbb{R}^{n}, a>0 \\
u(0 ; x)=f(x), x \in \mathbb{R}^{n}
\end{array}\right.
$$

where the function $f$ is continuous and bounded on $\mathbb{R}^{n}$.

We have the following classical result (see, for example, [23]).

Theorem 4.2. If the function $f$ is continuous and bounded on $\mathbb{R}^{n}$, then the Cauchy problem (4.24) has a unique solution in the class of continuous and bounded functions, which is given by the formula

$$
u(t, x)=\int_{\mathbb{R}^{n}} f(v) \frac{1}{(2 a \sqrt{\pi t})^{n}} \exp \left\{-\frac{|v-x|^{2}}{4 a^{2} t}\right\} d v, t>0, x \in \mathbb{R}^{n},
$$

and for each $x_{0} \in \mathbb{R}^{n} \lim _{t \rightarrow 0, x \rightarrow x_{0}} u(t, x)=f\left(x_{0}\right)$.

Note that in the literature the formula (4.25) is sometimes called the Poisson formula.

As a consequence, for the case of random initial condition we can state the next theorem.

Theorem 4.3. Let $\xi=\left\{\xi(x) ; x \in \mathbb{R}^{n}\right\}$ be an almost surely sample bounded and sample continuous process. Then with probability 1 the process

$$
u(t, x)=\int_{\mathbb{R}^{n}} \xi(v) \frac{1}{(2 a \sqrt{\pi t})^{n}} \exp \left\{-\frac{|v-x|^{2}}{4 a^{2} t}\right\} d v, t>0, x \in \mathbb{R}^{n},
$$

is a bounded and continuous solution to the Cauchy problem (4.24)with the random initial condition $u(0, x)=$ $\xi(x), x \in R^{n}$.

From Theorem 4.3 and Lemma 4.1 we obtain the following theorem.

Theorem 4.4. Consider the Cauchy problem (4.24) with the random initial condition $u(0, x)=\xi(x), x \in \mathbb{R}^{n}$, where $\xi$ is a stochastic process belonging to an Orlicz space $L_{U}(\Omega)$ and satisfying the conditions of Theorem 3.4 (or Theorem 3.5, that is, $U(x)=|x|^{p}, x \in \mathbb{R}, p \geq 1$ ). Then with probability 1, there exists the solution $u(t, x)$ of the 
Cauchy problem (4.24), given by the formula (4.26), such that $\sup _{t \geq 0, x \in \mathbb{R}}|u(t, x)|<\infty$ with probability 1, and

$$
\mathrm{P}\left\{\sup _{t \geq 0, x \in \mathbb{R}}|u(t, x)|>\varepsilon\right\} \leq \sum_{k \in \mathbb{Z}} Z_{k}(\varepsilon),
$$

where $Z_{k}(\varepsilon)=\left(U\left(\frac{\varepsilon}{R\left(t_{0 k}\right)}\right)\right)^{-1}$, as defined in (3.14) (or $Z_{k}(\varepsilon)=\left(\frac{R\left(\bar{t}_{0 k}\right)}{\varepsilon}\right)^{p}$, as defined in (3.17), for the case $\left.U(x)=|x|^{p}, x \in \mathbb{R}, p \geq 1\right)$.

We should note that, in fact, due to the particular "good" properties of the heat kernel, the properties of the solution to the Cauchy problem (4.24) can also be analyzed under the weaker conditions on the stochastic process $\xi$, but we will not go further in this direction here.

Next we can consider kernels $p$ coming from equations with fractional derivatives.

Consider the following fractional equation

$$
\frac{\partial^{\nu} u}{\partial t^{\nu}}=\lambda^{2} \frac{\partial^{2} u}{\partial x^{2}}, x \in \mathbb{R}, t>0,0<\nu \leq 2,
$$

where the fractional derivative $\frac{\partial^{\nu} u}{\partial t^{\nu}}$ is understood in the Caputo sense. This equation is called the fractional diffusion equation. For $\nu=1$ it reduces to the heat equation.

The fundamental solution to (4.27), with $0<\nu \leq 1$ and $u(0, x)=\delta(x)$ is given by (see, for example, [20]):

$$
p(t, x)=\frac{1}{2 \lambda t^{\nu / 2}} W_{-\frac{\nu}{2}, 1-\frac{\nu}{2}}\left(-\frac{|x|}{\lambda t^{\nu / 2}}\right), t>0, x \in \mathbb{R}
$$

where $W_{\alpha, \beta}$ is the Wright function

$$
W_{\alpha, \beta}=\sum_{k=0}^{\infty} \frac{x^{k}}{k ! \Gamma(\alpha k+\beta)}, \alpha>-1, \beta>0, x \in \mathbb{R} .
$$

For $\nu=\frac{1}{2}$ the solution is

$$
p_{1 / 2}(t, x)=\int_{0}^{\infty} \frac{e^{-\frac{x^{2}}{2 s}}}{\sqrt{2 \pi s}} \frac{e^{-\frac{s^{2}}{2 t}}}{\sqrt{2 \pi t}} d s
$$

which coincides with the probability density function of the composition of two independent Brownian motions $B_{1}\left(\left|B_{2}(t)\right|\right), t>0$ (see, [18]).

It was shown in [19] that for $\nu=\frac{1}{2^{n}}$ the solution to (4.27) for $\lambda^{2}=2^{1 / 2^{n}-2}$ gives the probability density function of the iterated process $B_{1}\left(\mid B_{2}\left(\left|B_{3}\left(\cdots\left(\left|B_{n+1}(t)\right| \cdots\right) \mid\right)\right|\right), t>0\right.$, with $B_{j}$ 's being independent Brownian motions, and this solution is of the following form:

$$
p_{1 / 2^{n}}(t, x)=\int_{0}^{\infty} \cdots \int_{0}^{\infty} \frac{e^{-\frac{x^{2}}{2 z_{1}}}}{\sqrt{2 \pi z_{1}}} \frac{e^{-\frac{z_{1}^{2}}{2 z_{2}}}}{\sqrt{2 \pi z_{2}}} \cdots \frac{e^{-\frac{z_{n}^{2}}{2 t}}}{\sqrt{2 \pi t}} d z_{1} \cdots d z_{n} .
$$

We mention just one more interesting density function

$$
p_{d}\left(t, x_{1}, x_{2}, \ldots, x_{d}\right)=2 \int_{0}^{\infty} \frac{e^{-\frac{w^{2}}{16 \lambda^{4} t}}}{\sqrt{2 \pi\left(8 \lambda^{4} t\right)}} \prod_{k=1}^{d} \frac{e^{-\frac{x_{k}^{2}}{2 w}}}{\sqrt{2 \pi w}} d w
$$

which is the probability density function of the vector process $\left(B_{1}(|B(t)|), \ldots, B_{d}(|B(t)|)\right)^{\prime}$, and represents a solution to the fractional equation

$$
\frac{\partial^{\frac{1}{2}} u}{\partial t^{\frac{1}{2}}}=\lambda^{2} \sum_{j=1}^{d} \frac{\partial^{2} u}{\partial x_{j}^{2}}, x_{j} \in \mathbb{R}, j=1, \ldots, d, t>0
$$


(see [19]).

One can observe the clear connection of the solutions (4.28)-(4.30) presented above with the Gaussian densities giving the heat kernel for the case of non-fractional equation. Note that for the cases $\nu=\frac{1}{3}$ and $\nu=\frac{2}{3}$ situation is different, and solutions to (4.27) are represented by means of the Airy functions.

By using (4.28), (4.29) or (4.30) as kernels in the integral (4.19) we obtain the models of processes, which can be treated as solutions to equation (4.27) with $\nu=1 / 2, \nu=1 /\left(2^{n}\right)$ or equation (4.31) correspondingly, with the random initial condition given by an almost surely bounded and continuous process $\xi$.

In all the above situations, if we take as initial condition a stochastic process $\xi$ from an Orlicz space $L_{U}(\Omega)$, which satisfies the conditions of Theorem 3.4 (or Theorem 3.5), then the corresponding process $u$, which represents the solution, is bounded and the distribution of its supremum coincides with that of $\xi$, and therefore, can be estimated by the expressions given in Theorem 3.4 (or in Theorem 3.5).

\section{Examples}

We present now several examples of processes satisfying the conditions of Theorem 3.5 and Corollary 3.6.

Example 5.1. Let $X(t)=c(t) \sum_{m=1}^{\infty} \xi_{m} \phi_{m}(t), t \in \mathbb{R}$, where $\xi_{m} \in L_{p}(\Omega), p \geq 1, m \in \mathbb{N}$, are independent random variables. Let $B_{k}=\left[a_{k}, a_{k+1}\right], k \in \mathbb{Z}, \mathbb{R}=\bigcup_{k \in \mathbb{Z}} B_{k}$ and functions $(c(t), t \in \mathbb{R})$ and $\left(\phi_{m}(t), t \in \mathbb{R}\right), m \geq 1$, satisfy the following assumptions:

$c(t)>0, t \geq 0$, is a monotone function, $c(t)=c(-t)$ and for $k \in \mathbb{Z}$ :

$$
\begin{aligned}
\sup _{t \in B_{k}}|c(t)| & \leq f_{k}, \\
\sup _{\substack{t, s \in B_{k},|t-s| \leq h}}|c(t)-c(s)| & \leq Z_{k} \sigma(h), h \geq 0, \\
\sup _{t \in B_{k}}\left|\phi_{m}(t)\right| & \leq d_{m}, \\
\sup _{\substack{t, s \in B_{k},|t-s| \leq h}}\left|\phi_{m}(t)-\phi_{m}(s)\right| & \leq r_{m} \sigma(h), m \geq 1, h \geq 0,
\end{aligned}
$$

where $(\sigma(h), h \geq 0)$ is a monotonically increasing continuous function such that $\sigma(0)=0,\left(r_{m}, m \in \mathbb{N}\right),\left(d_{m}, m \in\right.$ $\mathbb{N}),\left(Z_{k}, k \in \mathbb{Z}\right)$ are some numerical sequences.

We have

$$
\sup _{t \in B_{k}}\|X(t)\|_{p} \leq \sup _{t \in B_{k}}\left\{|c(t)| \sum_{m=1}^{\infty}\left\|\xi_{m}\right\|_{p}\left|\phi_{m}(t)\right|\right\} \leq f_{k} \sum_{m=1}^{\infty}\left\|\xi_{m}\right\|_{p} d_{m}=f_{k} A_{1},
$$

where $A_{1}=\sum_{m=1}^{\infty}\left\|\xi_{m}\right\|_{p} d_{m}$.

Suppose that $A_{1}<\infty$. Then $\sup _{t \in B_{k}}\|X(t)\|_{p} \leq f_{k} A_{1}<\infty$.

Next we evaluate the norm of the increments:

$$
\begin{aligned}
\|X(t)-X(s)\|_{p}=\| \sum_{m=1}^{\infty} \xi_{m}(c(t) & \left.\phi_{m}(t)-c(s) \phi_{m}(s)\right) \|_{p} \\
\leq & \sum_{m=1}^{\infty}\left\|\xi_{m}\right\|_{p}\left(\left|c(t) \| \phi_{m}(t)-\phi_{m}(s)\right|\right)+\sum_{m=1}^{\infty}\left\|\xi_{m}\right\|_{p}\left(\left|\phi_{m}(s) \| c(t)-c(s)\right|\right)
\end{aligned}
$$


and

$$
\begin{aligned}
& \sup _{|t-s| \leq h, t, s \in B_{k}}\|X(t)-X(s)\|_{p} \leq \sum_{m=1}^{\infty}\left\|\xi_{m}\right\|_{p}\left[r_{m} \sigma(h) f_{k}+d_{m} Z_{k} \sigma(h)\right]= \\
& =\sigma(h)\left[f_{k} \sum_{m=1}^{\infty}\left\|\xi_{m}\right\|_{p} r_{m}+Z_{k} \sum_{m=1}^{\infty}\left\|\xi_{m}\right\|_{p} d_{m}\right] \leq \sigma(h)\left[f_{k} A_{2}+Z_{k} A_{1}\right],
\end{aligned}
$$

where we suppose that $A_{2}=\sum_{m=1}^{\infty}\left\|\xi_{m}\right\|_{p} r_{m}<\infty$. Therefore, in the conditions of Corollary 3.6, $r\left(B_{k}\right)=$ $f_{k} A_{2}+Z_{k} A_{1}$.

Let for all $\varepsilon>0$ :

$$
\int_{0}^{\varepsilon}\left|\frac{1}{2 \sigma^{(-1)}(u)}+1\right|^{1 / p} d u<\infty
$$

then

$$
\begin{aligned}
& \int_{0}^{\sigma\left(\sup _{t \in B_{k}}\left|t_{0 k}-t\right|\right)}\left|\frac{a_{k+1}-a_{k}}{2 \sigma^{(-1)}(u)}+1\right|^{1 / p} d u \\
& \leq \max \left(\left|a_{k+1}-a_{k}\right|, 1\right) \int_{0}^{\sigma\left(\left|a_{k+1}-a_{k}\right|\right)}\left|\frac{1}{2 \sigma^{(-1)}(u)}+1\right|^{1 / p} d u .
\end{aligned}
$$

From (5.32) and (5.34) we obtain

$$
R\left(t_{0 k}\right) \leq A_{1} f_{k}+\frac{f_{k} A_{2}+Z_{k} A_{1}}{\theta(1-\theta)} \max \left(\left|a_{k+1}-a_{k}\right|, 1\right) \int_{0}^{\sigma\left(\left|a_{k+1}-a_{k}\right|\right)}\left|\frac{1}{2 \sigma^{(-1)}(u)}+1\right|^{1 / p} d u=: R_{k} .
$$

If

$$
\sum_{k \in \mathbb{Z}} R_{k}^{p}<\infty
$$

then

$$
\mathrm{P}\left\{\sup _{t \in \mathbb{R}}|X(t)|>\varepsilon\right\} \leq \frac{1}{\varepsilon^{p}} \sum_{k \in \mathbb{Z}} R_{k}^{p} .
$$

and the process $X$ is almost surely sample bounded and continuous on $\mathbb{R}$.

Example 5.2. Let in the conditions of Example 5.1, assume $\phi_{m}(t)=\sin \lambda_{m} t$, where $0<\lambda_{1}<\lambda_{2} \ldots, \lambda_{m} \rightarrow \infty$, $m \rightarrow \infty$.

Then $\left|\phi_{m}(t)\right| \leq 1=d_{m}$, and we suppose $A_{1}=\sum_{m=1}^{\infty}\left\|\xi_{m}\right\|_{p}<\infty$.

We have:

$$
\left|\phi_{m}(t)-\phi_{m}(s)\right|=\left|\sin \lambda_{m} t-\sin \lambda_{m} s\right| \leq 2\left|\sin \frac{\lambda_{m} t-\lambda_{m} s}{2}\right|,
$$

and by taking into account that $|\sin x| \leq|x|^{\alpha}$ for $0<\alpha \leq 1$, we obtain that

$$
2\left|\sin \frac{\lambda_{m} t-\lambda_{m} s}{2}\right| \leq \lambda_{m}^{\alpha} 2^{1-\alpha}|t-s|^{\alpha} .
$$

Therefore, in the conditions of Example 5.1, $r_{m}=2^{1-\alpha} \lambda_{m}^{\alpha}, \sigma(h)=h^{\alpha}, A_{2}=2^{1-\alpha} \sum_{m=1}^{\infty}\left\|\xi_{m}\right\|_{p} \lambda_{m}^{\alpha}$, and we suppose that $A_{2}<\infty$.

Now let $a_{0}=0, a_{1}=1, a_{-k}=-a_{k}$, and $0<a_{k+1}-a_{k} \leq 1, k>0, B_{k}=\left[a_{k}, a_{k+1}\right], k \in \mathbb{Z}$, and $\mathbb{R}=\bigcup_{k \in \mathbb{Z}} B_{k}$. Let $c(t)>0, c(-t)=c(t), t \in \mathbb{R}$, and

$$
c(t)=\left\{\begin{array}{l}
\frac{1}{t^{\beta}}, t>1, \beta>1 \\
1,0 \leq t \leq 1
\end{array}\right.
$$


Then $\sup _{t \in B_{k}} c(t)=\frac{1}{a_{k}^{\beta}}=f_{k}, k>0$. Since $\sigma^{(-1)}(u)=u^{1 / \alpha}, h \geq 0$, then we obtain

$$
\begin{aligned}
\int_{0}^{\varepsilon}\left|\frac{1}{2 \sigma^{(-1)}(u)}+1\right|^{1 / p} d u= & \int_{0}^{\varepsilon}\left|\frac{1}{2 u^{1 / \alpha}}+1\right|^{1 / p} d u \\
& \leq \int_{0}^{\varepsilon} \frac{1}{2^{1 / p} u^{1 / p \alpha}} d u+\varepsilon=\frac{1}{2^{1 / p}} \frac{\varepsilon^{1-\frac{1}{p \alpha}}}{1-\frac{1}{p \alpha}}+\varepsilon
\end{aligned}
$$

for $\frac{1}{p \alpha}<1$, that is, for $\alpha>\frac{1}{p}$. We can evaluate

$$
\begin{aligned}
\int_{0}^{\sigma\left(a_{k+1}-a_{k}\right)}\left|\frac{1}{2 \sigma^{(-1)}(u)}+1\right|^{1 / p} d u & \leq \frac{1}{2^{1 / p}}\left(a_{k+1}-a_{k}\right)^{\alpha-\frac{1}{p}}\left(1-\frac{1}{p \alpha}\right)^{-1}+\left(a_{k+1}-a_{k}\right)^{\alpha} \\
& \leq \frac{1}{2^{1 / p}}\left(1-\frac{1}{p \alpha}\right)^{-1}+1 .
\end{aligned}
$$

Next, for $1<t<s, k>1$, we obtain:

$$
\begin{aligned}
\sup _{t, s \in B_{k},|t-s| \leq h}(c(t)-c(s))= & \sup _{t, s \in B_{k},|t-s| \leq h}\left(\frac{1}{t^{\beta}}-\frac{1}{s^{\beta}}\right) \leq \sup _{t, s \in B_{k},|t-s| \leq h} \frac{s^{\beta}-t^{\beta}}{a_{k}^{2 \beta}} \\
= & \sup _{t, s \in B_{k},|t-s| \leq h} \frac{1}{a_{k}^{2 \beta}} \int_{t}^{s} \frac{1}{\beta} u^{\beta-1} d u \leq \sup _{t, s \in B_{k},|t-s| \leq h}(s-t) \frac{a_{k+1}^{\beta-1}}{\beta a_{k}^{2 \beta}} \\
& \leq h \frac{a_{k+1}^{\beta-1}}{\beta a_{k}^{2 \beta}} \leq h^{\alpha} \frac{1}{\beta}\left(1+\frac{1}{a_{k}}\right)^{\beta-1} \frac{1}{a_{k}^{\beta+1}} \leq h^{\alpha} \frac{2^{\beta-1}}{\beta} \frac{1}{a_{k}^{\beta+1}} .
\end{aligned}
$$

Therefore, $Z_{k}=\frac{2^{\beta-1}}{\beta a_{k}^{\beta+1}}$, and we arrive at the following bound:

$$
R_{k} \leq A_{1} \frac{1}{a_{k}^{\beta}}+4\left[\frac{1}{a_{k}^{\beta}} A_{2}+\frac{1}{\beta a_{k}^{\beta+1}} 2^{\beta-1} A_{1}\right]\left(\frac{1}{2^{1 / p}}\left(1-\frac{1}{p \alpha}\right)^{-1}+1\right)=: \hat{R_{k}},
$$

since at $\theta=\frac{1}{2}$ we have the maximum of $\frac{1}{\theta(1-\theta)}$. We have also $\hat{R}_{k}=\hat{R}_{-k}$. Now we find the condition for the convergence

$$
\sum_{k \in \mathbb{Z}} \hat{R}_{k}^{p}<\infty
$$

We have the following estimate:

$$
\begin{aligned}
\hat{R}_{k} \leq \frac{1}{a_{k}^{\beta}}\left(A_{1}+4 A_{2}\left(\frac{1}{2^{1 / p}}+1\right)\right)+\frac{1}{\beta a_{k}^{\beta+1}} 2^{\beta-1} & A_{1}\left(\frac{1}{2^{1 / p}}+1\right) \leq \\
& \leq \frac{1}{a_{k}^{\beta}}\left(A_{1}+4 A_{2}\left(\frac{1}{2^{1 / p}}+1\right)+2^{\beta-1} A_{1}\left(\frac{1}{2^{1 / p}}+1\right)\right)=: \widetilde{R}_{k},
\end{aligned}
$$

therefore, the convergence of the series $\sum_{k \in \mathbb{Z}} \hat{R}_{k}^{p}$ is implied by the convergence of $\sum_{k \in \mathbb{Z}} \frac{1}{a_{k}^{\beta p}}$.

In the conditions of Corollary 3.6 and Example 5.1, $r\left(B_{k}\right)=f_{k} A_{2}+Z_{k} A_{1} \leq \frac{4 A_{2}}{a_{k}^{\beta}}\left(\frac{1}{2} \beta+1\right)$. With the imposed condition on $\beta$, the series $\sum_{k \in \mathbb{Z}} \frac{1}{a_{k}^{\beta}}$ is convergent, as well as $\sum_{k \in \mathbb{Z}} \frac{1}{a_{k}^{\beta p}}<\infty$, since $\frac{1}{a_{k}^{\beta}} \geq \frac{1}{a_{k}^{\beta p}}$.

Therefore, if $\sum_{k \in \mathbb{Z}} \frac{1}{a_{k}^{\beta}}<\infty, A_{1}<\infty, A_{2}<\infty$, then the process $X(t)=c(t) \sum_{m=1}^{\infty} \xi_{m} \sin \lambda_{m} t, t \in \mathbb{R}$, satisfies the conditions of Theorem 4.3, hence, with probability 1 the process $X$ is sample bounded and continuous and the following estimate for the distribution of its supremum holds:

$$
\mathrm{P}\left\{\sup _{t \in \mathbb{R}}|X(t)|>\varepsilon\right\} \leq \frac{1}{\varepsilon^{p}} \sum_{k \in \mathbb{Z}} \widetilde{R}_{k}^{p} .
$$


Example 5.3. Let $X(t), t \in \mathbb{R}$, be a process from the space $L_{2}(\Omega), \mathrm{E} X(t)=0, \mathrm{E} X(t) X(s)=\int_{\mathbb{R}} f(v, t) f(v, s) d v$, where the function $f(v, t), v, t \in \mathbb{R}$, is such that

$$
\int_{\mathbb{R}} f^{2}(v, t) d v<\infty
$$

We have $\mathrm{E} X^{2}(t)=\int_{\mathbb{R}} f^{2}(v, t) d v$ and $\mathrm{E}|X(t)-X(s)|^{2}=\int_{\mathbb{R}}|f(v, t)-f(v, s)|^{2} d v$.

Let under the conditions of Corollary 3.6, $B_{k}=\left[a_{k}, a_{k+1}\right], k \in \mathbb{Z}$, and $\mathbb{R}=\bigcup_{k \in \mathbb{Z}} B_{k}$, where $a_{0}=0, a_{-k}=$ $-a_{k}, a_{k}<a_{k+1}$ for $k \in \mathbb{N}$.

Suppose that the following conditions hold:

$$
|f(v, t)| \leq g\left(\left|a_{k}\right|\right) \hat{f}(v), t \in B_{k},|f(v, t)-f(v, s)| \leq \sigma(|t-s|) Z\left(\left|a_{k}\right|\right) \hat{f}(v), t, s \in B_{k},
$$

where $\sigma(h), h>0$, is a monotonically increasing continuous function, $\sigma(0)=0$, and for $\varepsilon>0$

$$
\int_{0}^{\varepsilon}\left|\frac{1}{2 \sigma^{(-1)}(u)}+1\right|^{1 / 2} d u<\infty
$$

$Z(v), g(v), v>0$, are monotonically nondecreasing functions, and the function $\hat{f}(v)>0, v \in \mathbb{R}$, is such that $\int_{\mathbb{R}} \hat{f}^{2}(v) d v<\infty$.

Denote $\|\hat{f}\|_{2}=\left(\int_{\mathbb{R}} \hat{f}^{2}(v) d v\right)^{1 / 2}$.

We have

$$
\left(\mathrm{E}|X(t)-X(s)|^{2}\right)^{1 / 2} \leq \sigma(|t-s|) Z\left(\left|a_{k}\right|\right)\|\hat{f}\|_{2}, t, s \in B_{k},
$$

therefore, in terms of Corollary 3.6, $r\left(\hat{B_{k}}\right)=Z\left(\left|a_{k}\right|\right)\|\hat{f}\|_{2}$, and we obtain the bound:

$$
R\left(t_{0 k}\right) \leq \hat{R}\left(t_{0 k}\right):=g\left(\left|a_{k}\right|\right)\|\hat{f}\|_{2}+\frac{1}{\theta(1-\theta)} Z\left(\left|a_{k}\right|\right)\|\hat{f}\|_{2} \int_{0}^{\sigma\left(\left|a_{k+1}-a_{k}\right|\right)}\left|\frac{a_{k+1}-a_{k}}{2 \sigma^{(-1)}(v)}+1\right|^{1 / 2} d v .
$$

If the series

$$
\sum_{k \in \mathbb{Z}}\left(\hat{R}\left(t_{0 k}\right)\right)^{2}
$$

is convergent, then in view of Corollary 3.6, the process $X$ is sample continuous with probability $1, \sup _{t \in \mathbb{R}}|X(t)|<$ $\infty$ and for $\varepsilon>0$

$$
\mathrm{P}\left\{\sup _{t \in \mathbb{R}}|X(t)|>\varepsilon\right\} \leq \frac{1}{\varepsilon^{2}} \sum_{k \in \mathbb{Z}}\left(\hat{R}\left(t_{0 k}\right)\right)^{2} .
$$

Thus, from Theorem 4.4, we have that the process

$$
u(t, x)=\int_{\mathbb{R}} X(v) \frac{1}{2 a \sqrt{\pi t}} \exp \left\{-\frac{(v-x)^{2}}{4 a^{2} t}\right\} d v
$$

is a bounded solution to the Cauchy problem (4.24) and the following inequality holds for $\varepsilon>0$

$$
\mathrm{P}\left\{\sup _{x \in \mathbb{R}, t>0}|u(t, x)|>\varepsilon\right\} \leq \frac{1}{\varepsilon^{2}} \sum_{k \in \mathbb{Z}}\left(\hat{R}\left(t_{0 k}\right)\right)^{2} .
$$

Example 5.4. Let in the conditions of Example 5.3, $f(v, t)=f(v) \sin \frac{|v|}{1+|t|}, v, t \in \mathbb{R}$, where $f(v) \geq 0, v \in \mathbb{R}$, is a symmetric function.

We have, similar to Example 5.3,

$$
|f(v, t)|=|f(v)|\left|\sin \frac{|v|}{1+|t|}\right| \leq \frac{|v|^{\alpha}}{(1+|t|)^{\alpha}} f(v), \text { for } 0<\alpha \leq 1,
$$


and

$$
\begin{aligned}
|f(v, t)-f(v, s)|=f(v)\left|\sin \frac{|v|}{1+|t|}-\sin \frac{|v|}{1+|s|}\right| \leq 2 f(v)\left|\sin \frac{|v|}{2} \frac{|s-t|}{(1+|t|)(1+|s|)}\right| \leq \\
\leq 2 f(v) \frac{|v|^{\beta}}{2^{\beta}} \frac{|s-t|^{\beta}}{(1+|t|)^{\beta}(1+|s|)^{\beta}}, \text { for } 0<\beta \leq 1 .
\end{aligned}
$$

Then for $B_{k}$ defined as in Example 5.3,

$$
\sup _{t \in B_{k}}\left(\int_{\mathbb{R}}|f(v, t)|^{2} d v\right)^{1 / 2} \leq \frac{1}{\left(1+\left|a_{k}\right|\right)^{\alpha}}\left(\int_{\mathbb{R}}|v|^{2 \alpha} f^{2}(v) d v\right)^{1 / 2},
$$

and

$$
\sup _{t, s \in B_{k},|t-s| \leq h}\left(\int_{\mathbb{R}}|f(v, t)-f(v, s)|^{2} d v\right)^{1 / 2} \leq \frac{2^{1-\beta} h^{\beta}}{\left(1+\left|a_{k}\right|\right)^{2 \beta}}\left(\int_{\mathbb{R}}|v|^{2 \beta} f^{2}(v) d v\right)^{1 / 2} .
$$

Therefore, in the notations of Example 5.3, $\sigma(h)=h^{\beta}, g\left(\left|a_{k}\right|\right)=\frac{1}{\left(1+\left|a_{k}\right|\right)^{\alpha}}, Z\left(\left|a_{k}\right|\right)=\frac{2^{1-\beta}}{\left(1+\left|a_{k}\right|\right)^{2 \beta}}$, and in the conditions of Corollary 3.6, $r\left(B_{k}\right)=Z\left(\left|a_{k}\right|\right) f(v)|v|^{\beta}$.

If $\int_{\mathbb{R}}|v|^{2 \alpha} f^{2}(v) d v<\infty$ and $\int_{\mathbb{R}}|v|^{2 \beta} f^{2}(v) d v<\infty$, then

$$
\begin{gathered}
\int_{0}^{\varepsilon}\left|\frac{1}{2 \sigma^{(-1)}(u)}+1\right|^{1 / 2} d u=\int_{0}^{\varepsilon}\left(\frac{1}{2 u^{1 / \beta}}+1\right)^{1 / 2} d u \leq \frac{1}{\sqrt{2}} \frac{\varepsilon^{1-\frac{1}{2 \beta}}}{1-\frac{1}{2 \beta}}+\varepsilon, \\
\int_{0}^{\sigma\left(\mid a_{k+1}-a_{k}\right) \mid}\left|\frac{1}{2 u^{1 / \beta}}+1\right|^{1 / 2} d u \leq \frac{\sqrt{2} \beta}{2 \beta-1}\left(a_{k+1}-a_{k}\right)^{\beta-1 / 2}+\left(a_{k+1}-a_{k}\right)^{\beta}<\infty
\end{gathered}
$$

for $\beta>\frac{1}{2}$. We obtain:

$$
\begin{aligned}
R\left(t_{0 k}\right) \leq R_{k}:=g\left(\left|a_{k}\right|\right)\left(\int_{\mathbb{R}} \mid\right. & \left.\left.v\right|^{2 \alpha} f^{2}(v) d v\right)^{1 / 2}+ \\
& +4 Z\left(\left|a_{k}\right|\right)\left(\int_{\mathbb{R}}|v|^{2 \beta} f^{2}(v) d v\right)^{1 / 2}\left(\frac{\sqrt{2} \beta}{2 \beta-1}\left(a_{k+1}-a_{k}\right)^{\beta-1 / 2}+\left(a_{k+1}-a_{k}\right)^{\beta}\right) .
\end{aligned}
$$

Let us suppose that $\alpha=\beta>\frac{1}{2}$ and $\int_{\mathbb{R}}|v|^{2 \alpha} f^{2}(v) d v<\infty$. We can always choose $a_{k}$ such that the series

$$
\sum_{k \in \mathbb{Z}} R_{k}^{2}
$$

is convergent. For example, we can take $a_{k}=e^{k}$.

Then it follows from Corollary 3.6 that with probability 1 the process $X$ is sample continuous, $\sup _{t \in \mathbb{R}}|X(t)|<$ $\infty$ and for $\varepsilon>0$

$$
\mathrm{P}\left\{\sup _{t \in \mathbb{R}}|X(t)|>\varepsilon\right\} \leq \frac{1}{\varepsilon^{2}} \sum_{k \in \mathbb{Z}} R_{k}^{2} .
$$

Therefore, for the process $u(t, x)=\int_{\mathbb{R}} X(v) \frac{1}{2 a \sqrt{\pi t}} \exp \left\{-\frac{(v-x)^{2}}{4 a^{2} t}\right\} d v$ we have the same bound for $\varepsilon>0$ :

$$
\mathrm{P}\left\{\sup _{x \in \mathbb{R}, t>0}|u(t, x)|>\varepsilon\right\} \leq \frac{1}{\varepsilon^{2}} \sum_{k \in \mathbb{Z}} R_{k}^{2} .
$$




\section{Acknowledgments}

We are grateful to the reviewer for valuable remarks and suggestions, which helped us to improve the paper significantly.

\section{REFERENCES}

1. R.J. Adler, An introduction to continuity, extrema and related topics for general Gaussian processes, Lecture Notes-Monograph Series. Institute of Mathematical Statistics, Hayward, Vol. 12, 160 p., 1990.

2. R.J. Adler, The Geometry of Random Fields, Society for Industrial and Applied Mathematics; Siam ed. edition, 184 p., 2009.

3. R.J. Adler, J.E. Taylor, Random Fields and Geometry, Springer Monographs in Mathematics, 448 p., 2007.

4. V. V. Buldygin, Yu. V. Kozachenko, Metric Characterization of Random Variables and Random Processes, American Mathematical Society, Providence, RI, 257 p., 2000.

5. I. Dariychuk, Yu. Kozachenko, M. Perestyuk, Random processes from Orlicz spaces, K.: Zoloti Lytavry, 212 p., 2011. (in Ukrainian)

6. M. Dozzi, Yu. Kozachenko, Yu. Mishura, K. Ralchenko, Asymptotic growth of trajectories of multifractional Brownian motion with statistical applications to drift parameter estimation, Statistical Inference for Stochastic processes, vol. 21(1), pp. 21-52, 2018.

7. R. M. Dudley, The sizes of compact subsets of Hilbert space and continuity of Gaussian processes, Journal of Functional Analysis, vol. 1, pp. 290-330, 1967.

8. R.M. Dudley, Uniform Central Limit Theorems, Cambridge Studies in Advanced Mathematics, vol. 63. Cambridge University Press, Cambridge, 452 p., 1999.

9. X. Fernique, Régularité des trajectoires des fonctions aléatoires gaussiennes, In: Ecole d'Eté de Probabilités de Saint-Flour, IV-1974. Lecture Notes in Math., vol. 480, pp. 1-96. Springer, Berlin 1975.

10. Yu. Kozachenko, A. Melnikov, Yu. Mishura, On drift parameter estimation in models with fractional Brownian motion, Statistics: A Journal of theoretical and applied statistics, vol. 49(1), pp. 35-62, 2015.

11. Yu. Kozachenko, E. Orsingher, L. Sakhno, O. Vasylyk, Estimates for functionals of solutions to Higher-Order Heat-Type equations with random initial conditions, Journal of Statistical Physics, vol. 72(6), pp. 1641-1662, 2018.

12. Yu. Kozachenko, M. Perestyuk, On the uniform convergence of wavelet expansions of random processes from Orlicz spaces of random variables. I., Ukrainian Math. J., vol. 59, No. 12, pp. 1850-1869, 2007.

13. Yu. Kozachenko, M. Perestyuk, On the uniform convergence of wavelet expansions of random processes from Orlicz spaces of random variables. II., Ukrainian Math. J., vol. 60, No. 6, pp. 876-900, 2008.

14. Yu.V. Kozachenko, A.I. Slyvka-Tylyshchak, On the increase rate of random fields from space $S_{u}(\Omega)$ on unbounded domains, Statistics, Optimization and Information Computing, vol. 2, No. 2, pp. 79-92, 2014.

15. Yu.V. Kozachenko, K.I. Veresh, The heat equation with random initial conditions from Orlicz spaces, Theory Probab. Math. Stat., vol. 80, pp. 71-84, 2010.

16. M. Ledoux, M. Talagrand, Probability in a Banach Space: Isoperimetry and Processes, Ergebnisse der Mathematik und ihrer Grenzgebiete (3), vol. 23. Springer, Berlin, 482 p., 1991.

17. M. Ledoux, Isoperimetry and Gaussian Analysis, In: Lecture Notes in Mathematics, Vol. 1648, pp. 165-294, Springer, 1996.

18. E. Orsingher, L. Beghin, Time-fractional equations and telegraph processes with Brownian time, Probab. Theory Relat. Fields, vol. 128, pp.141-160, 2004.

19. E. Orsingher, L. Beghin, Fractional diffusion equations and processes with randomly-varying time, Ann. Prob., vol. 37(1), pp. 206$249,2009$.

20. I. Podlubny, Fractional Differential Equations. Mathematics in Science and Engineering 198, Academic Press, San Diego, CA., 340 p., 1999.

21. M. Talagrand, The Generic Chaining. Upper and Lower Bounds of Stochastic Processes. Springer Monographs in Mathematics, Springer, Berlin, 222 p., 2005.

22. M. Talagrand, Upper and Lower Bounds for Stochastic Processes. Modern Methods and Classical Problems, Springer-Verlag: Berlin, Heidelberg, 626 p., 2014.

23. D.V. Widder, The Heat Equation, Academic Press: New York, San Francisco, London, 267 p., 1975. 